\title{
Reflection of Obliquely Incident Guided Waves by an Edge of a Plate
}

\author{
Arief Gunawan and Sohichi Hirose \\ Department of Mechanical and Environmental Informatics, Tokyo Institute of Technology, Tokyo 152-8552, Japan
}

This paper analyzes the edge-reflection problem of obliquely incident guided waves in a plate. The generalized guided-wave theories in a plate, including the orthogonality of modes and the mode-decomposition method are summarized. The edge-reflection problem is solved on the basis of the mode-decomposition method. Some numerical results are presented and compared to experimental results. [doi:10.2320/matertrans.I-MRA2007852]

(Received September 8, 2006; Accepted March 6, 2007; Published May 25, 2007)

Keywords: guided waves, plate, edge reflection, oblique incidence, mode-decomposition method

\section{Introduction}

Guided waves are widely applied in nondestructive evaluations to detect defects in a thin plate. Because guided waves can propagate along a plate structure with small attenuation, guided wave nondestructive evaluations are able to scan a wide range of the plate fast without moving a transducer during inspection. The propagation of guided waves in a plate with obstacles is, however, very complicated because the interaction between guided waves and obstacles causes mode conversion which involves not only propagating modes but also an infinite number of nonpropagating modes. ${ }^{1-3)}$ To increase the ability of guided wave nondestructive evaluations, knowledge on scattering behaviors of guided waves by various obstacles is beneficial. In particular, reflections of guided waves by an edge of a plate are essential because the reflections are always involved in practice.

Reflection of a normally incident antiplane wave ( $\mathrm{SH}$ wave) by an edge is quite a simple problem since no mode conversion occurs. On the other hand, reflection of a normally incident inplane wave (Lamb wave) by an edge is more complicated because it causes mode conversion involving an infinite number of Lamb wave modes. The edge-reflection problem of the normally incident Lamb wave has been analyzed by various methods, such as the variational analysis method, ${ }^{4,5)}$ the collocation method, ${ }^{6,7)}$ the method of projection, ${ }^{8)}$ the finite element method in a time domain, $\left.{ }^{6}\right)$ the finite element method combined with the modal expansion technique, ${ }^{9,10)}$ the hybrid method of the boundary element method and the normal-mode expansion technique, ${ }^{11)}$ the hybrid finite element and boundary element formulation, ${ }^{10)}$ and the mode-exciting method. ${ }^{2)}$ The reflection of the normally incident Lamb wave by the beveled edge has been analyzed by Wilkie-Chancellier et al. ${ }^{7)}$ and by Galan and Abascal. ${ }^{10)}$ Experimental studies on the reflection of the normally incident Lamb wave have been carried out by Morvan $^{6)}$ and Wilkie-Chancellier et al. ${ }^{13)}$

This study analyzes the reflection of obliquely incident guided waves by a plate edge. For normally incident guided waves to the plate edge, Lamb waves and $\mathrm{SH}$ waves are separately analyzed even in the reflection process, whereas Lamb waves and $\mathrm{SH}$ waves are coupled each other in the case of oblique incidence. In the reflection problems of obliquely incident guided waves by the plate edge, mode conversions between Lamb and $\mathrm{SH}$ wave modes have to be taken into account. In the following, the generalized orthogonality satisfied by the guided wave modes is first derived. Based on the generalized orthogonality, the mode decomposition method of an elastodynamic field into guided wave modes is proposed. The reflection problems of obliquely incident guided waves by an edge are then solved semi-analytically by using the mode decomposition method. Experiments are carried out to verify the analytical results.

\section{Generalized Theories of Guided Wave Modes in a Plate}

\subsection{Displacements and stresses of guided waves}

Let us consider a homogeneous, isotropic and linearly elastic plate with the thickness $2 h$ as shown in Fig. 1. On the assumption that the traction free condition is given on the upper and lower surfaces of the plate and the wave field in the plate is time harmonic with the circular frequency $\omega$, there are two types of guided waves which can propagate in the plate, i.e., Lamb waves with inplane motions and $\mathrm{SH}$ waves with antiplane motions. The dispersive relations of symmetric and antisymmetric Lamb wave modes ${ }^{1-3)}$ and $\mathrm{SH}$ wave modes $^{2,3)}$ are given by

$$
\frac{\tan (q h)}{\tan (p h)}+\frac{4 k^{2} p q}{\left(q^{2}-k^{2}\right)^{2}}=0,
$$

(symmetric Lamb waves)

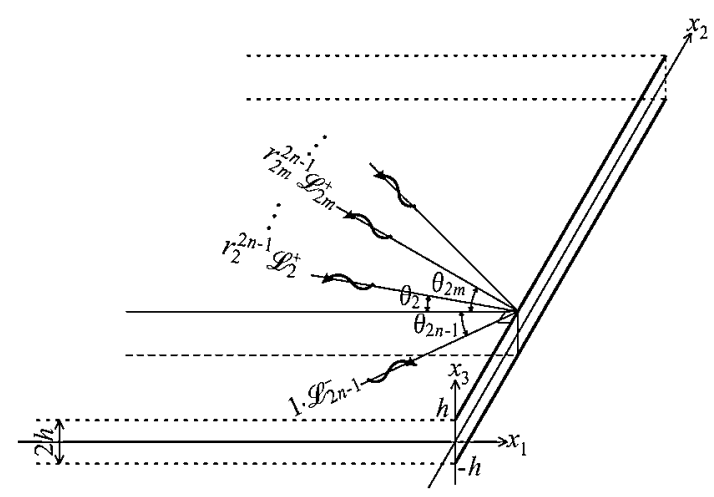

Fig. 1 Reflection of an obliquely incident guided wave by an edge. 


$$
\begin{aligned}
& \frac{\tan (q h)}{\tan (p h)}+\frac{\left(q^{2}-k^{2}\right)^{2}}{4 k^{2} p q}=0, \\
&(\text { antisymmetric Lamb waves) } \\
& q h-\frac{n \pi}{2}=0,
\end{aligned}
$$

$$
\text { (SH waves) }
$$

respectively, where $k$ denotes the wave number. In eqs. (1), (2), and (3),

$$
p^{2}=\frac{\omega^{2}}{c_{L}^{2}}-k^{2}, \quad q^{2}=\frac{\omega^{2}}{c_{T}^{2}}-k^{2},
$$

where $c_{L}$ and $c_{T}$ are the velocities of the longitudinal and transverse waves, respectively. $n$ in eq. (3) is a non-negative integer and takes even and odd numbers for symmetric and antisymmetric $\mathrm{SH}$ wave modes, respectively. As numerical examples, the dispersion curves of Lamb wave and $\mathrm{SH}$ wave modes for the steel with $c_{L}=5940 \mathrm{~m} / \mathrm{s}$ and $c_{T}=3200 \mathrm{~m} / \mathrm{s}$, which was used in our experiments, are shown in Figs. 2(a) and (b), respectively. $S_{n}$ and $A_{n}$ in Fig. 2(a) and $S H_{n}$ in Fig. 2(b) denote the symmetric and antisymmetric Lamb wave modes and $\mathrm{SH}$ wave modes, respectively, of the $n$th order $(n=0,1,2, \ldots)$. Solid and dotted curves denote the guided waves with pure real and pure imaginary wavenumbers, respectively. Dashed and double dashed curves denote the real parts and the imaginary parts, respectively, of the wavenumbers of the guided waves with complex wavenumbers.

Taking the propagation direction in the $x_{1}$-axis, the displacement $\boldsymbol{U}$ of a guided wave can be expressed as

$$
\boldsymbol{U}=A \hat{\boldsymbol{U}} e^{I\left(k x_{1}-\omega t\right)},
$$

where $\hat{U}$ is the displacement in the frequency-wavenumber

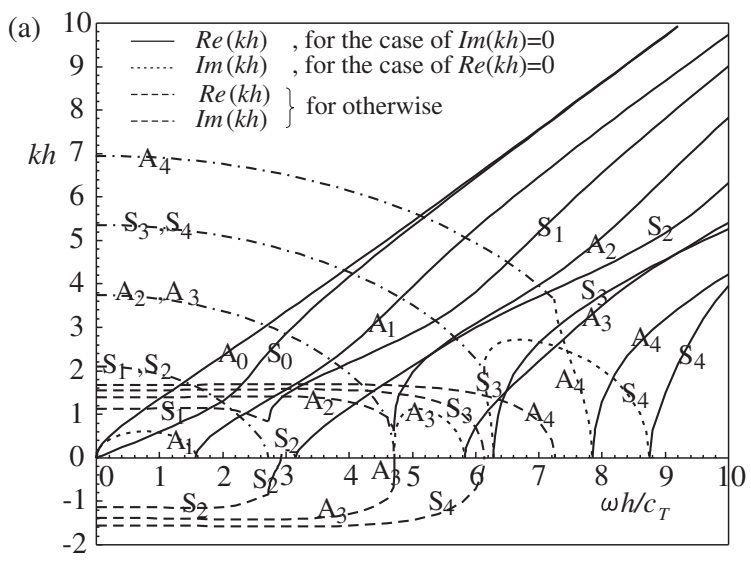

(b)

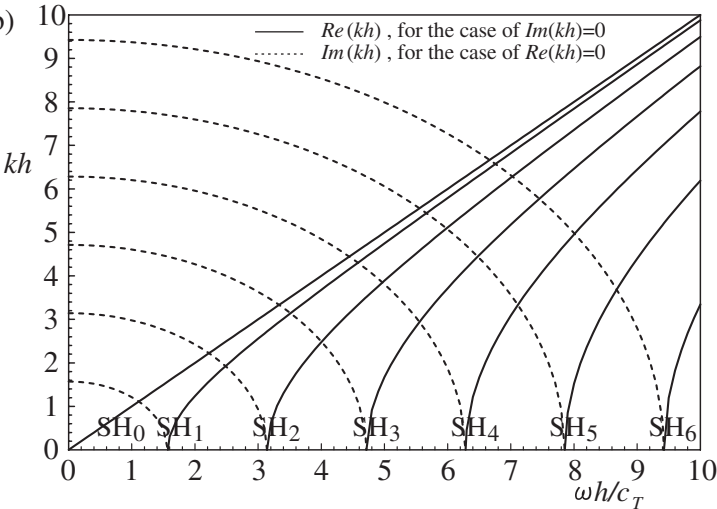

Fig. 2 Dispersion curves for (a) Lamb wave modes and (b) SH wave modes.

domain and is a function of $x_{3}$ only, $I=\sqrt{-1}$, and $A$ is an arbitrary constant. The expressions of $\hat{U}$ are given by

$$
\begin{aligned}
& \hat{U}_{1}=\operatorname{IAk}\left[\frac{\cos \left(p x_{3}\right)}{\sin (p h)}+\frac{2 p q}{k^{2}-q^{2}} \frac{\cos \left(q x_{3}\right)}{\sin (q h)}\right] \\
& \hat{U}_{2}=0 \\
& \text { for symmetric Lamb wave modes, } \\
& \hat{U}_{3}=-A p\left[\frac{\sin \left(p x_{3}\right)}{\sin (p h)}-\frac{2 k^{2}}{k^{2}-q^{2}} \frac{\sin \left(q x_{3}\right)}{\sin (q h)}\right] \\
& \hat{U}_{1}=I A k\left[\frac{\sin \left(p x_{3}\right)}{\cos (p h)}+\frac{2 p q}{k^{2}-q^{2}} \frac{\sin \left(q x_{3}\right)}{\cos (q h)}\right] \\
& \hat{U}_{2}=0 \\
& \text { for antisymmetric Lamb wave modes, } \\
& \left.\hat{U}_{3}=A p\left[\frac{\cos \left(p x_{3}\right)}{\cos (p h)}-\frac{2 k^{2}}{k^{2}-q^{2}} \frac{\cos \left(q x_{3}\right)}{\cos (q h)}\right]\right\} \\
& \left.\begin{array}{l}
\hat{U}_{1}=\hat{U}_{3}=0 \\
\hat{U}_{2}=A k \cos \left(q x_{3}\right)
\end{array}\right\} \text { for symmetric SH wave modes, and }
\end{aligned}
$$

In case that the guided wave travels in the direction $\theta$ with respect to the $x_{1}$ direction, the displacement $\boldsymbol{u}$ of the guided wave is represented by

$$
\boldsymbol{u}=A \hat{\boldsymbol{u}} e^{I\left(\eta x_{1}+\xi x_{2}-\omega t\right)},
$$

where 


$$
\begin{aligned}
& \left\{\begin{array}{l}
\hat{u}_{1}=\frac{\eta}{k} \hat{U}_{1}-\frac{\xi}{k} \hat{U}_{2}, \\
\hat{u}_{2}=\frac{\xi}{k} \hat{U}_{1}+\frac{\eta}{k} \hat{U}_{2}, \\
\hat{u}_{3}=\hat{U}_{3},
\end{array}\right. \\
& \xi=k \sin (\theta), \quad \text { and }
\end{aligned}
$$

In eq. (12), the sign \pm of the root is chosen as follows. When $\Im(k)=0$, the sign of the root is chosen so that the signs \pm of $\Re(\eta)$ and $\Re(k)$ are the same, where $\mathfrak{R}$ and $\mathfrak{s}$ mean the real and imaginary parts, respectively. When $\Im(k) \neq 0$, the sign of the root is chosen so that the signs \pm of $\Im(\eta)$ and $\Im(k)$ are the same. Note that when $\xi$ are fixed, there is a one-to-one relation between $k$ and $\eta$.

The stress components $\tau_{i j}$ can be evaluated by substituting eq. (10) into the stress-displacement relation

$$
\tau_{i j}=\rho\left(c_{L}^{2}-2 c_{T}^{2}\right) u_{k, k} \delta_{i j}+\rho c_{T}^{2}\left(u_{i, j}+u_{j, i}\right),
$$

where $\rho$ is the density and $\delta_{i j}$ is the Kronecker delta.

\subsection{Generalized orthogonality of guided wave modes}

Let $\mathscr{L}_{1}, \mathscr{L}_{2}, \ldots$ be the guided wave modes including the four types of guided wave modes, i.e., the symmetric/ antisymmetric Lamb/SH wave modes, with the wavenumber $k_{1}, k_{2}, \ldots$. Suppose that all modes $\mathscr{L}_{m}$ travel in such directions that their wavenumbers in the $x_{2}$-direction are equal to $\xi$, where $\xi$ is a real value. Using eq. (12), we can determine $\eta_{1}, \eta_{2}, \ldots$ uniquely for all modes. It is assumed that if $\mathscr{L}_{m}$ and $\mathscr{L}_{n}$ are the modes of the same type and $m \neq n$, then we have $k_{m} \neq k_{n}$ and, consequently, $\eta_{m} \neq \eta_{n}$. Note that when $k_{m}$ is complex-valued, $\eta_{m}$ is always complex-valued. $\eta_{m}$ can, however, be complex-valued even if $k_{m}$ is real, i.e., when $\left|k_{m}\right|<|\xi|$. For real and complex-valued $\eta_{m}, \mathscr{L}_{m}$ are propagating and nonpropagating modes, respectively.

We now consider two modes $\mathscr{L}_{m}$ and $\mathscr{L}_{n}$ that have the displacement and stress fields $[\boldsymbol{u}, \boldsymbol{\tau}]$ and $[\boldsymbol{v}, \boldsymbol{\sigma}]$, respectively, where

$$
\begin{aligned}
& \boldsymbol{u}=\tilde{\boldsymbol{u}} e^{-I \omega t}=\hat{\boldsymbol{u}} e^{I\left(\eta_{m} x_{1}+\xi x_{2}-\omega t\right)}, \boldsymbol{\tau}=\tilde{\boldsymbol{\tau}} e^{-I \omega t}=\hat{\boldsymbol{\tau}} e^{I\left(\eta_{m} x_{1}+\xi x_{2}-\omega t\right)}, \\
& \boldsymbol{v}=\tilde{\boldsymbol{v}} e^{-I \omega t}=\hat{\boldsymbol{v}} e^{I\left(\eta_{n} x_{1}+\xi x_{2}-\omega t\right)}, \boldsymbol{\sigma}=\tilde{\boldsymbol{\sigma}} e^{-I \omega t}=\hat{\boldsymbol{\sigma}} e^{I\left(\eta_{n} x_{1}+\xi x_{2}-\omega t\right)} .
\end{aligned}
$$

Following Auld's work, ${ }^{3)}$ the complex reciprocity identity is applied to two wave fields of eq. (14) to obtain the generalized orthogonality of guided wave modes in a plate as follows:

$$
\left(\eta_{m}-\eta_{n}^{*}\right) P_{m n}^{\xi}=0,
$$

where the superscript * means the complex conjugate and

$$
P_{m n}^{\xi}=\frac{I \omega}{4} \int_{-h}^{h}\left(\hat{u}_{i} \hat{\sigma}_{1 i}^{*}-\hat{v}_{i}^{*} \hat{\tau}_{1 i}\right) d x_{3} .
$$

Similarly to the derivation shown in our previous paper, ${ }^{12}$ it can be shown that $P_{m n}^{\xi}$ has the physical meaning as follows:

$$
\mathscr{P}_{1}\left[\mathscr{L}_{m}+\mathscr{L}_{n}\right]=\mathscr{P}_{1}\left[\mathscr{L}_{m}\right]+\mathscr{P}_{1}\left[\mathscr{L}_{n}\right]+\mathfrak{R}\left(2 P_{m n}^{\xi}\right),
$$

where $\mathscr{P}_{1}$, defined as eq. (18), denotes the power per unit length passing through a plane which is perpendicular to the $x_{1}$-axis.

$$
\mathscr{P}_{1}\left[\mathscr{L}_{m}\right]=\Re\left[-\frac{I \omega}{2} \int_{-h}^{h} \tilde{u}_{i}^{*} \tilde{\tau}_{1 i} d x_{3}\right] .
$$

Note that the properties of $P_{m n}^{\xi}$ is similar to $P_{m n}$ in our previous paper. ${ }^{12)}$

In particular case that $\xi=0$, we have $\eta_{m}=k_{m}$ and the orthogonality shown in eq. (15) reduces into the orthogonality shown in our previous paper. ${ }^{12)}$ If $\eta_{m}=\eta_{n}^{*}$, the direct substitution of eqs. (10) and (13) into eq. (16) yields the following relation between $P_{m n}^{\xi}$ and $P_{m n}^{0}\left(=\left.P_{m n}^{\xi}\right|_{\xi=0}\right)$ as

$$
P_{m n}^{\xi}=\frac{\eta_{m}}{k_{m}} P_{m n}^{0}, \quad \text { if } \quad \eta_{m}=\eta_{n}^{*} .
$$

Hereafter we use the notation $Q_{m}^{\xi}$ to refer $P_{m n}^{\xi}$ for which $\eta_{m}=\eta_{n}^{*}$.

\subsection{Normalization and mode decomposition}

The normalization and the mode decomposition can be performed similarly to those in our previous paper. ${ }^{12)}$ Here we only show a summary of the normalization and the mode decomposition.

The normalized displacement $\overline{\boldsymbol{u}}$ of a propagating guided wave mode $\mathscr{L}_{m}$ is defined as

$$
\overline{\boldsymbol{u}}=\frac{\boldsymbol{u}}{\sqrt{\left|\mathscr{P}_{1}\left[\mathscr{L}_{m}\right]\right|}},
$$

where $\boldsymbol{u}$ is the displacement of mode $\mathscr{L}_{m}$.

If $\boldsymbol{u}$ and $\boldsymbol{v}$ are displacements of conjugate nonpropagating guided wave modes $\mathscr{L}_{m}$ and $\mathscr{L}_{n}$, respectively, the normalized displacements $\overline{\boldsymbol{u}}$ and $\overline{\boldsymbol{v}}$ are defined as

$$
\overline{\boldsymbol{u}}=\frac{\boldsymbol{u}}{\sqrt{\left|P_{m n}^{\xi}\right|}}, \quad \overline{\boldsymbol{v}}=\frac{\boldsymbol{v}}{\sqrt{\left|P_{m n}^{\xi}\right|}},
$$

where $P_{m n}^{\xi}$ is defined in eq. (16).

Any arbitrary time-harmonic elastodynamic field $\mathscr{E}$ in the plate which satisfies the traction-free boundary condition on the top and bottom surfaces and has the wavenumber $\xi$ in the $x_{2}$-direction can be decomposed into guided wave modes as follows:

$$
\mathscr{E}=\sum_{m=1}^{\infty} A_{m} \mathscr{L}_{m}
$$

The amplitudes $A_{m}$ of the guided wave modes $\mathscr{L}_{m}$ can be found by

$$
A_{m}=\frac{\mathscr{E} \odot \mathscr{L}_{\gamma(m)}}{Q_{m}^{\xi}} .
$$

In eq. (23), $\gamma(m)$ is the index satisfying the conjugate relation of $\eta_{\gamma(m)}=\eta_{n}^{*}$ and the operator ' $\odot$ ' relates two time-harmonic elastodynamic fields $\mathscr{E}$ and $\mathscr{E}^{\prime}$ to a scalar value as follows:

$$
\mathscr{E} \odot \mathscr{E}^{\prime} \equiv \frac{I \omega}{4} \int_{-h}^{h}\left(\tilde{u}_{i} \tilde{\sigma}_{1 i}^{*}-\tilde{v}_{i}^{*} \tilde{\tau}_{1 i}\right) d x_{3},
$$

where $\mathscr{E}=\left[\tilde{\boldsymbol{u}} e^{-i \omega t}, \tilde{\boldsymbol{\tau}} e^{-i \omega t}\right]$ and $\mathscr{E}^{\prime}=\left[\tilde{\boldsymbol{v}} e^{-i \omega t}, \tilde{\boldsymbol{\sigma}} e^{-i \omega t}\right]$.

\section{Statement of the Problem}

In this section, the statement of the reflection problem of obliquely incident guided waves by an edge of a plate is presented. In the previous section, the guided wave modes in 
$\mathscr{L}_{n}(n=1,2, \ldots)$ include the modes travelling in both $+x_{1}$ and $-x_{1}$ directions. In the edge-reflection problem, it is preferable to distinguish the modes travelling in the $+x_{1}$ and $-x_{1}$ directions, which represent the incident and reflected waves with respect to the edge, respectively. Hereafter, without losing the orthogonal property, we assume that $\mathscr{L}_{n}$ $(n=1,2, \ldots)$ are ordered as follows:

- $\mathscr{L}_{2 m-1}(m=1,2, \ldots)$ are the modes which travel in $+x_{1}$ direction, i.e., $\Re\left(\eta_{2 m-1}\right)>0$ if $\Im\left(\eta_{2 m-1}\right)=0$, or $\Im\left(\eta_{2 m-1}\right)>0$ if $\Im\left(\eta_{2 m-1}\right) \neq 0$.

- $\mathscr{L}_{2 m}(m=1,2, \ldots)$ are the modes which travel in $-x_{1}$ direction, i.e., $\Re\left(\eta_{2 m}\right)<0$ if $\Im\left(\eta_{2 m}\right)=0$, or $\Im\left(\eta_{2 m}\right)<0$ if $\Im\left(\eta_{2 m}\right) \neq 0$.

Suppose that the guided wave mode $\mathscr{L}_{2 n-1}$ with a unit amplitude is obliquely incident to an edge of a plate with the angle $\theta_{2 n-1}$ and reflected waves of all guided wave modes $\mathscr{L}_{2 m}(m=1,2, \ldots)$ are generated with the amplitudes $r_{2 m}^{2 n-1}$ and travel obliquely with the angles $\theta_{2 m}$, as shown in Fig. 1. To satisfy the traction-free condition on the edge, the angles $\theta_{2 m}$ must follow the Snell's law:

$$
k_{2 m} \sin \left(\theta_{2 m}\right)=k_{2 n-1} \sin \left(\theta_{2 n-1}\right)=\xi, \quad(m=1,2, \ldots) .
$$

Note that when $k_{2 m}$ is a complex value or a real value less than $\xi$, the reflected mode $\mathscr{L}_{2 m}$ is a nonpropagating mode and cannot travel far from the edge.

In the reflection problem of guided waves, the reflection coefficients $r_{2 m}^{2 n-1}$ are the unknowns to be determined. Note that the coefficients $r_{2 m}^{2 n-1}$ are, in general, complex variables, and their square absolute value and argument represent the power in the $x_{1}$-direction and the phase shift, respectively.

\section{Semi-Analytical Method for Edge-Reflection Prob- lem}

In this section, the application of the mode decomposition to solve the reflection problem of the guided waves is presented.

Let $[\boldsymbol{v}, \boldsymbol{\sigma}]$ be the displacement and the stress of the total wave $\mathscr{E}$ in the reflection problem and $\left[\boldsymbol{u}^{j}, \boldsymbol{\tau}^{j}\right]$ be those of the guided wave mode $\mathscr{L}_{j}$. Since $\mathscr{E}$ is composed of the incident wave $\mathscr{L}_{2 n-1}$ with a unit amplitude and the reflected waves $\mathscr{L}_{2 m}(m=1,2, \ldots)$ with the amplutide of $r_{2 m}^{2 n-1}$, it is clear that

$$
\mathscr{E}=\mathscr{L}_{2 n-1}+\sum_{m=1}^{\infty} r_{2 m}^{2 n-1} \mathscr{L}_{2 m}
$$

Now we apply eq. (23) to decompose $\mathscr{E}$ into guided wave modes. According to eqs. (24) and (23), the amplitude $A_{2 j}$ of the $\mathscr{L}_{2 j}$ mode after the decomposition is

$$
\begin{aligned}
A_{2 j}= & \frac{\mathscr{E} \odot \mathscr{L}_{\gamma(2 j)}}{Q_{2 j}^{\xi}} \\
= & \frac{I \omega}{4 Q_{2 j}^{\xi}} \int_{-h}^{h}\left[\tilde{v}_{i}\left\{\tilde{\tau}_{1 i}^{\gamma(2 j)}\right\}^{*}-\left\{\tilde{u}_{i}^{\gamma(2 j)}\right\}^{*} \tilde{\sigma}_{1 i}\right] d x_{3} \\
& (j=1,2, \ldots) .
\end{aligned}
$$

From eq. (26), it is clear that $A_{2 j}$ must be equal to $r_{2 j}^{2 n-1}$. Hence we have

$$
\begin{array}{r}
r_{2 j}^{2 n-1}=\frac{I \omega}{4 Q_{2 j}^{\xi}} \int_{-h}^{h}\left[\tilde{v}_{i}\left\{\tilde{\tau}_{1 i}^{\gamma(2 j)}\right\}^{*}-\left\{\tilde{u}_{i}^{\gamma(2 j)}\right\}^{*} \tilde{\sigma}_{1 i}\right] d x_{3} \\
(j=1,2, \ldots) .
\end{array}
$$

Taking $x_{1}=0$, which is the location of the edge, we have the total stress components $\tilde{\sigma}_{1 i} \equiv 0$ because of the tractionfree condition on the edge. From eq. (26), the total displacement $\boldsymbol{v}$ can be represented as

$$
\boldsymbol{v}=\boldsymbol{u}^{2 n-1}+\sum_{m=1}^{\infty} r_{2 m}^{2 n-1} \boldsymbol{u}^{2 m}
$$

Furthermore, we have

$$
\tilde{v}_{i}\left\{\tilde{\tau}_{1 i}^{\gamma(2 j)}\right\}^{*}=\hat{v}_{i}\left\{\hat{\tau}_{1 i}^{\gamma(2 j)}\right\}^{*}
$$

at $x_{1}=0$. Substituting eqs. (28) and (29) and $\tilde{\sigma}_{1 i} \equiv 0$ into eq. (27), we have

$$
\begin{array}{r}
r_{2 j}^{2 n-1}=\frac{I \omega}{4 Q_{2 j}^{\xi}} \int_{-h}^{h}\left(\hat{u}_{i}^{2 n-1}+\sum_{m=1}^{\infty} r_{2 m}^{2 n-1} \hat{u}_{i}^{2 m}\right)\left\{\hat{\tau}_{1 i}^{\gamma(2 j)}\right\}^{*} d x_{3} \\
(j=1,2, \ldots) .
\end{array}
$$

or

$$
\begin{aligned}
& \sum_{m=1}^{\infty}\left[\int_{-h}^{h} \hat{u}_{i}^{2 m}\left\{\hat{\tau}_{1 i}^{\gamma(2 j)}\right\}^{*} d x_{3}-\frac{4 Q_{2 j}^{\xi}}{I \omega} \delta_{j m}\right] r_{2 m}^{2 n-1} \\
& \quad=-\int_{-h}^{h} \hat{u}_{i}^{2 n-1}\left\{\hat{\tau}_{1 i}^{\gamma(2 j)}\right\}^{*} d x_{3} \quad(j=1,2, \ldots) .
\end{aligned}
$$

Since there are an infinite number of guided wave modes, Equation (30) gives a system of linear equations with infinite numbers of equations $(j=1,2, \ldots)$ and unknown variables $r_{2 m}^{2 n-1}(m=1,2, \ldots)$. To solve the system of linear equations approximately, only a finite number of modes are considered in the numerical examples shown below. The finite number of modes are chosen so that they include all propagating modes and several nonpropagating modes with the smallest imaginary parts of $\eta$.

\section{Numerical Examples}

In the numerical examples shown below, 45 guided wave modes, composed of 30 Lamb wave modes and $15 \mathrm{SH}$ wave modes, are used in solving the system of linear equations (30). The accuracy of the numerical results is investigated by considering the power balance between the incident wave $\mathscr{L}_{n}^{-}$and all the reflected propagating modes $\mathscr{L}_{m}^{-}$as follows:

$$
\sum_{m}\left|r_{2 m}^{2 n-1}\right|^{2}=1
$$

All numerical results shown in the following are obtained with the residual error of eq. (31) less than 0.001 .

\subsection{Normal incidences}

As the first examples, the normal incidence $(\xi=0)$ of Lamb waves is considered. Figures 3(a)-(e) illustrate the reflection coefficients for the incident $A_{0}, A_{1}, S_{0}, S_{1}$, and $S_{2}$ waves, respectively. The abscissa and ordinate represent the nondimensional frequency $\omega h / c_{T}$ and the absolute value of reflection coefficients $\left|r_{2 m}^{2 n-1}\right|$, respectively. Note that since 

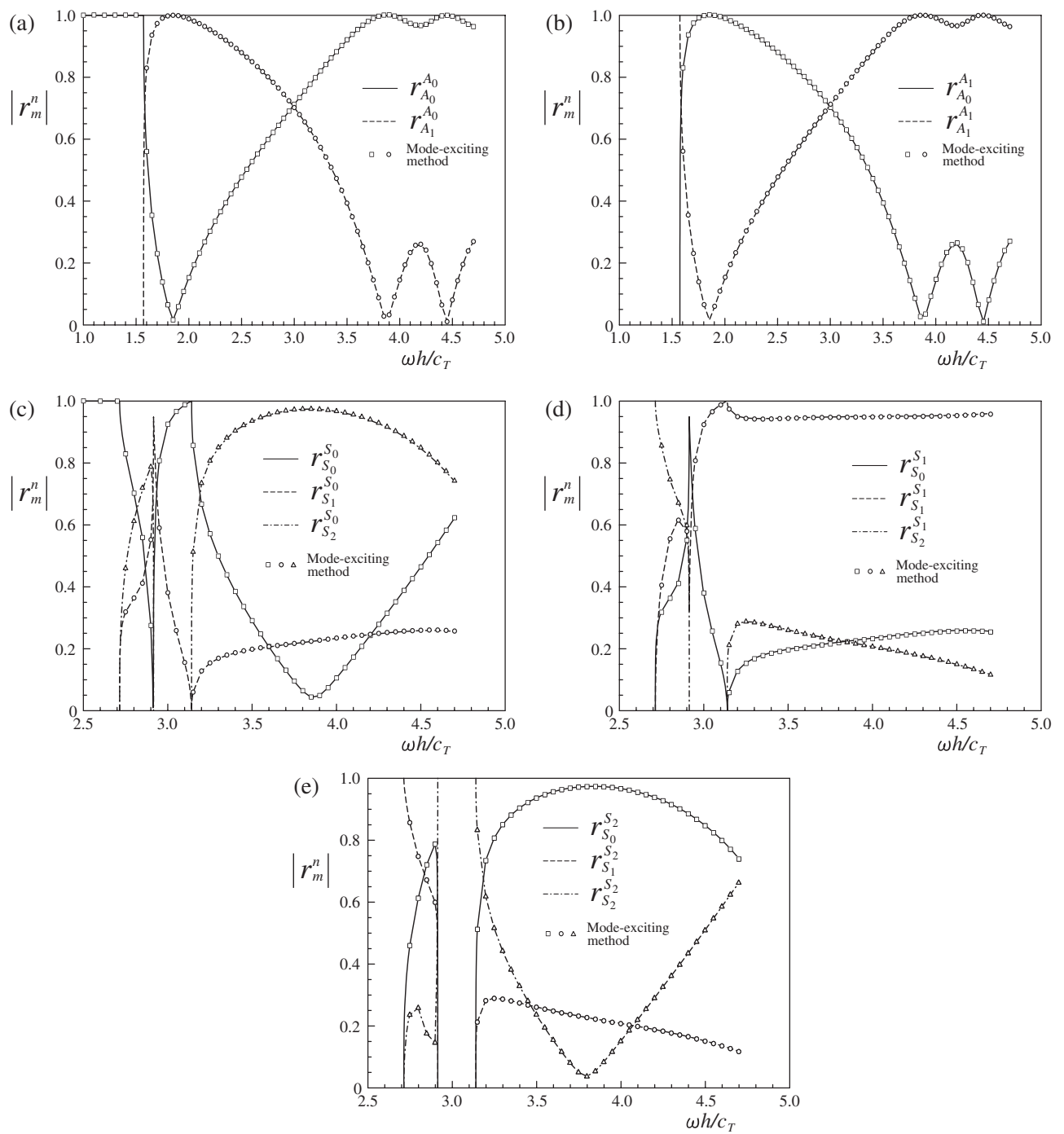

Fig. 3 Reflection coefficients for the normal incidence of (a) $A_{0}$ mode, (b) $A_{1}$ mode, (c) $S_{0}$ mode, (d) $S_{1}$ mode, and (e) $S_{2}$ mode.

the reflection problem is a symmetric problem in the thickness direction, the symmetric and antisymmetric modes are uncoupled each other. For comparison, the results obtained by the mode-exciting method ${ }^{12)}$ are also shown by symbols. Very good agreement is found between both results.

\subsection{Oblique incidences}

Next, some examples are shown for the oblique incidences of Lamb waves and $\mathrm{SH}$ waves. The frequency is fixed to $\omega h / c_{T}=1.96$, which is the value adopted in our experiments shown later. Similarly to the problem of normal incidence, the problem of oblique incidence is also a symmetric problem, and hence the symmetric and antisymmetric modes are uncoupled each other.

Figures 4(a) and (b) show the numerical results of the reflection coefficients for the $S H_{0}$ and $S_{0}$ modes incidences, respectively. The abscissa represents the wavenumber $\xi$ in the $x_{2}$-direction as well as the angles of propagation $\theta_{m}$ of the reflected propagating modes $\mathscr{L}_{m}^{+}$, where $\theta_{m}$ and $\xi$ are related by eq. (25). At $\omega h / c_{T}=1.96$, the wavenumbers $k_{S H_{0}} h$ and $k_{S_{0}} h$ of the $S H_{0}$ and $S_{0}$ modes are 1.96 and 1.28 , respectively. Since $\xi$ cannot be greater than the wavenumber of the incident wave, the results in Figs. 4(a) and (b) are shown in the range of $\xi<1.96$ and $\xi<1.28$, respectively.

When $\xi=0$, the $S H_{0}$ and $S_{0}$ modes are uncoupled each other in the reflection problem and only two symmetric modes of $S_{0}$ and $S_{0}$ exist at the frequency $\omega h / c_{T}=1.96$. Hence we have $\left|r_{S H_{0}}^{S H_{0}}\right|=\left|r_{S_{0}}^{S_{0}}\right|=1$ and $\left|r_{S_{0}}^{S H_{0}}\right|=\left|r_{S H_{0}}^{S_{0}}\right|=0$ at $\xi=0$. As $\xi$ increases, the $S H_{0}$ and $S_{0}$ modes become coupled each other so that $\left|r_{S_{0}}^{S H_{0}}\right|$ and $\left|r_{S H_{0}}^{S_{0}}\right|$ increase, whereas $\left|r_{S H_{0}}^{S H_{0}}\right|$ and $\left|r_{S_{0}}^{S_{0}}\right|$ decrease. Note that $\left|r_{S_{0}}^{S H_{0}}\right|=\left|r_{S H_{0}}^{S_{0}}\right|$ from the Betty's reciprocal theorem. It is also noticed that since the reflection coefficients must satisfy the power balance eq. (31) and at $\omega h / c_{T}=1.96$ the $S H_{0}$ and $S_{0}$ modes are the only symmetric modes, we obtain $\left|r_{S H_{0}}^{S H_{0}}\right|=\left|r_{S_{0}}^{S_{0}}\right|$. Total reflections of the $S_{0}$ and $S H_{0}$ modes are found for the incidences of $S H_{0}$ and $S_{0}$ modes, respectively at $\xi=0.896$. For $\xi>k_{S_{0}} h=1.28$, the $S_{0}$ mode becomes a nonpropagating mode and then only the $\mathrm{SH}_{0}$ mode is reflected for the $S H_{0}$ mode incidence. Thus we have $\left|r_{S H_{0}}^{S H_{0}}\right|=1$ for $1.28<\xi<1.96$, as shown in Fig. 4(a).

It is known that as $\omega h / c_{T} \rightarrow 0$, the $S_{0}$ mode is reduced to the longitudinal wave ${ }^{1)}$ with constant and zero values in $\hat{U}_{1}$ and $\hat{U}_{3}$, respectively [see eq. (6)]. Similarly, the $S H_{0}$ mode becomes the shear wave with the uniform $\hat{U}_{2}$, as $\omega h / c_{T} \rightarrow 0$ [see eq. (8)]. Considering the wave motions of $S_{0}$ and $\mathrm{SH}_{0}$ modes, it can be expected that the reflections of the $S_{0}$ and 

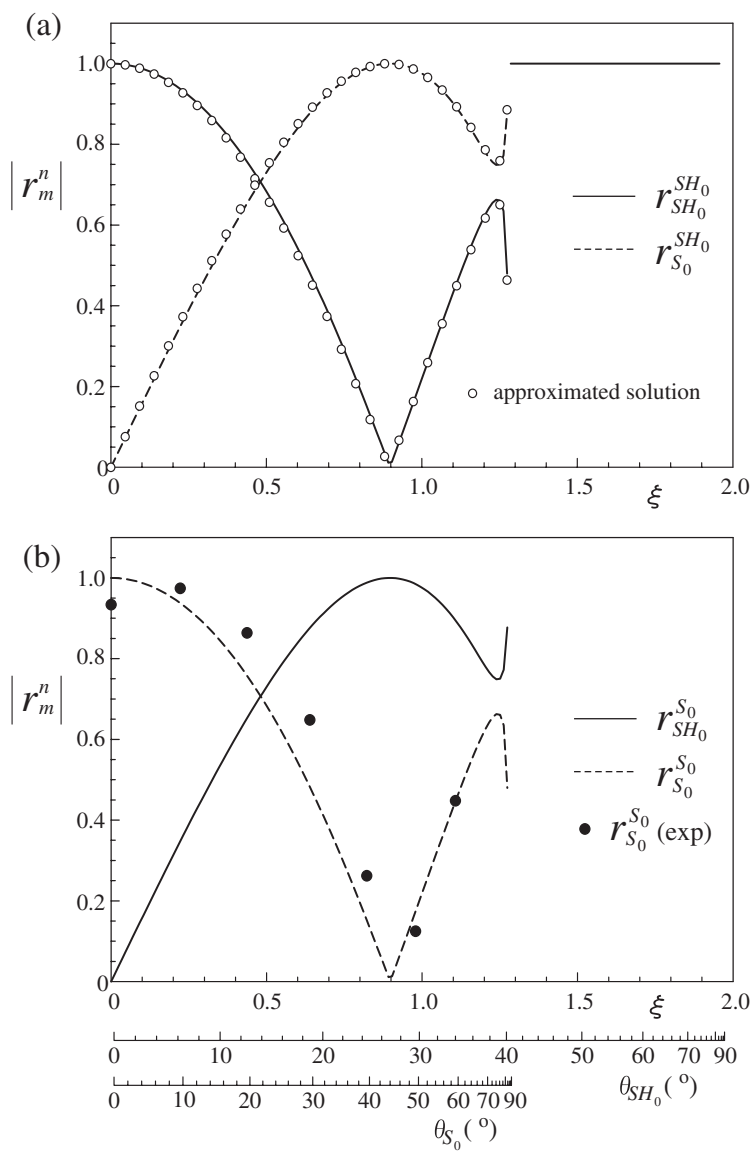

Fig. 4 Reflection coefficients as the function of $\xi$ for (a) $\mathrm{SH}_{0}$ mode and (b) $S_{0}$ mode incidences at the frequency $\omega h / c_{T}=1.96$.

$\mathrm{SH}_{0}$ modes at a free edge in the low frequency range are similar to the reflections of a P-wave and an SV-wave in a half-space, respectively. The reflection coefficients for the obliquely incident $S_{0}$ and $S H_{0}$ modes can, therefore, be approximated by using the amplitudes of the reflected $\mathrm{P}$ and SV waves in a half-space, respectively, as follows: (for example, see the book by Achenbach ${ }^{2)}$ )

$$
\begin{aligned}
& \left|r_{S H_{0}}^{S H_{0}}\right|=\left|r_{S_{0}}^{S_{0}}\right| \approx\left|\frac{\sin \left(2 \theta_{S_{0}}\right) \sin \left(2 \theta_{S H_{0}}\right)-\kappa^{2} \cos \left(2 \theta_{S H_{0}}\right)}{\sin \left(2 \theta_{S_{0}}\right) \sin \left(2 \theta_{S H_{0}}\right)+\kappa^{2} \cos \left(2 \theta_{S H_{0}}\right)}\right|, \\
& \left|r_{S H_{0}}^{S_{0}}\right|=\left|r_{S_{0}}^{S H_{0}}\right| \approx \sqrt{1-\left|r_{S_{0}}^{S_{0}}\right|^{2}},
\end{aligned}
$$

where $\kappa=k_{S H_{0}} / k_{S_{0}}$. The approximated values for $\left|r_{S H_{0}}^{S H_{0}}\right|$ and $\left|r_{S_{0}}^{S H_{0}}\right|$ are shown by circles in Fig. 4(a). Comparing the numerical and approximated values, it is found that eq. (32) can give good approximation for $\left|r_{S H_{0}}^{S H_{0}}\right|$ and $\left|r_{S_{0}}^{S H_{0}}\right|$ at $\omega h / c_{T}=1.96$.

The numerical results of the reflection coefficients for oblique incidences of $A_{0}, S H_{1}$, and $A_{1}$ modes are shown in Figs. 5(a), (b), and (c), respectively. At $\omega h / c_{T}=1.96$, the wavenumbers $k_{A_{0}} h, k_{S H_{1}} h$, and $k_{A_{1}} h$ of the $A_{0}, S H_{1}$, and $A_{1}$ modes are $2.32,1.18$, and 0.666 , respectively. As shown in Fig. 5(a), $\left|r_{A_{0}}^{A_{0}}\right|$ shows relatively small values for $\xi<k_{A_{1}} h$, increases to unity for $k_{A_{1}} h<\xi<k_{S H_{1}} h$ as $\xi$ increases, and becomes unity for $\xi>k_{S H_{1}} h$. In the case of $S H_{1}$ incidence, $\left|r_{S H_{1}}^{S H_{1}}\right|$ decreases at $\xi<k_{A_{1}} h$ and increases at $k_{A_{1}} h<\xi<$ $k_{S H_{1}} h$ as $\xi$ increases as shown in Fig. 5(b). As shown in Fig. 5(c), most power of the incident $A_{1}$ mode is reflected as
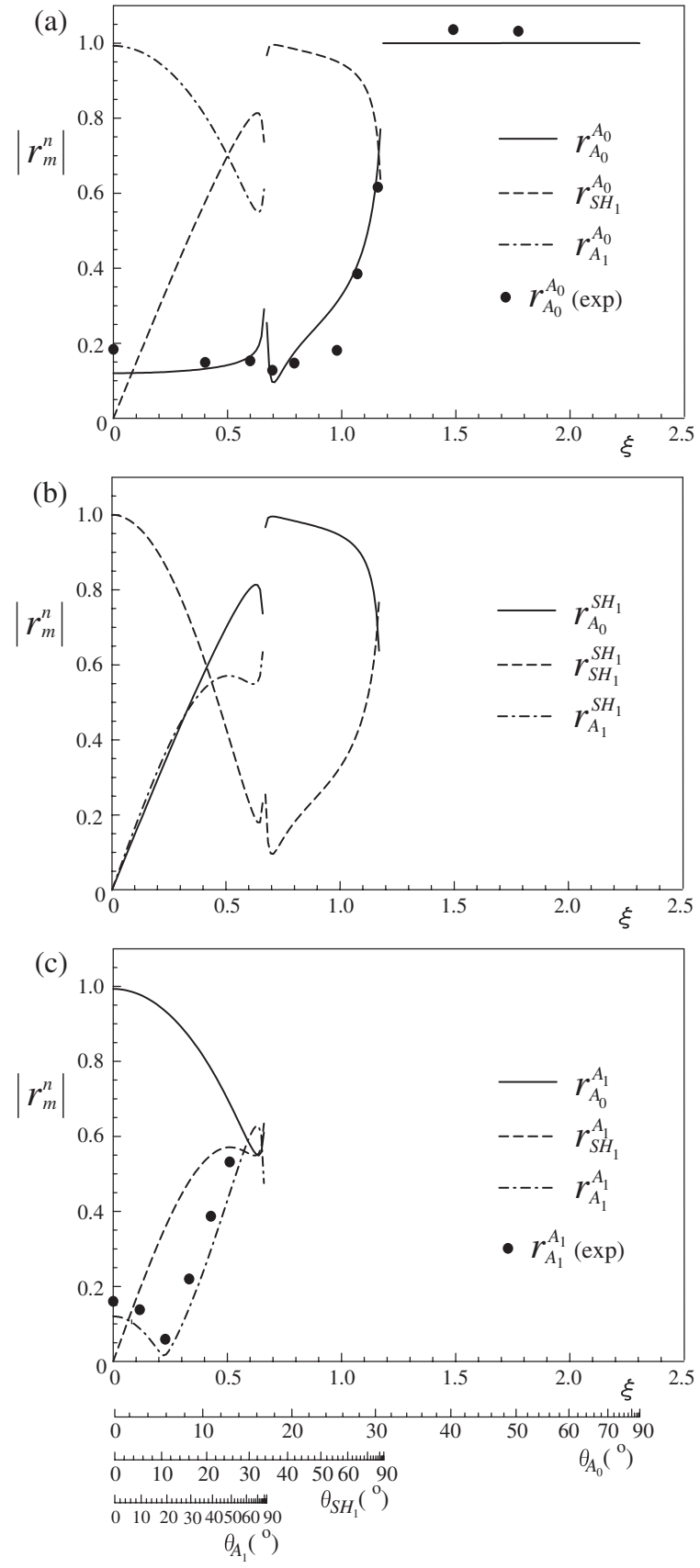

Fig. 5 Reflection coefficients as the function of $\xi$ for (a) $A_{0}$ mode, (b) $S H_{1}$ mode, and (c) $A_{1}$ mode incidences at the frequency $\omega h / c_{T}=1.96$.

the $A_{0}$ mode. In Fig. 5(c), it is also seen that $\left|r_{A_{1}}^{A_{1}}\right|$ shows a minimum value at $\xi=0.22$.

\section{Experiments}

Experiments for reflections of Lamb waves are carried out to verify the analytical results shown in the previous section. The $S_{0}, A_{0}$, and $A_{1}$ modes are chosen as incident waves.

The experimental setup is shown in Fig. 6(a). The function generator (Agilent 33250A) delivers the sinusoidal electrical signal with the frequency of $1 \mathrm{MHz}$ to the high power gated amplifier (Ritec GA-10000), which produces the 15-cycletoneburst signal. The 15-cycle-toneburst signal is sent directly to the transducer made by Japan Probe Co. with 

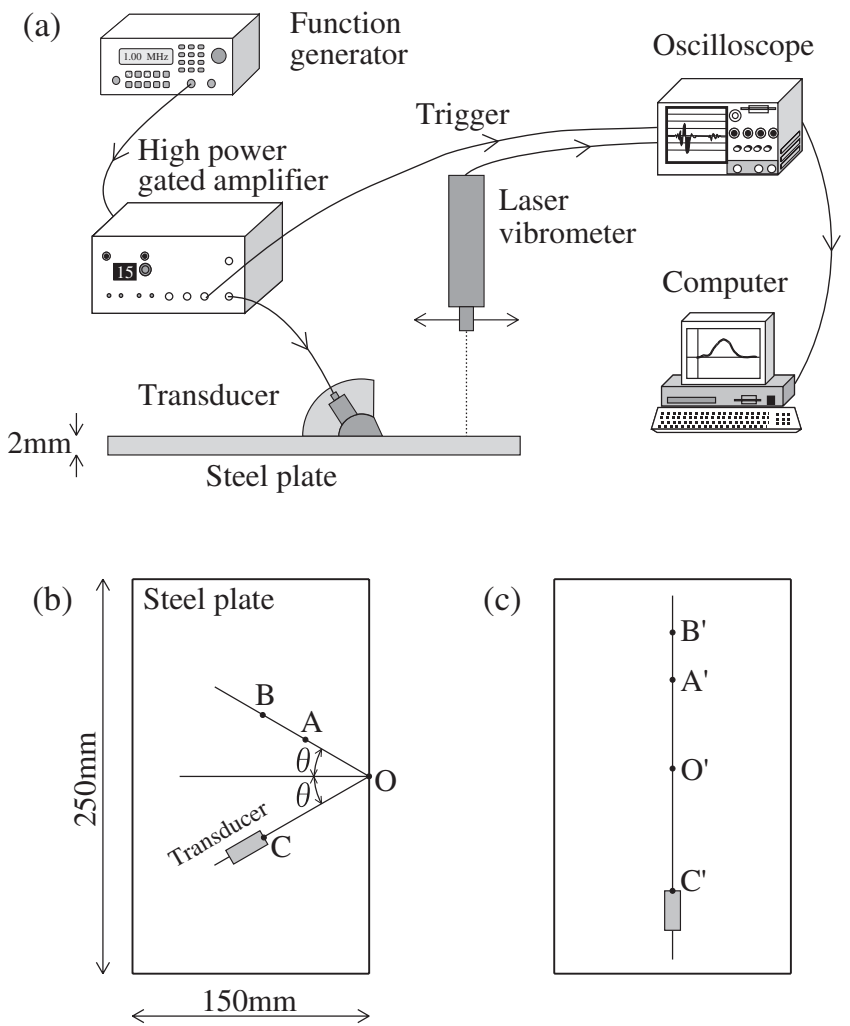

Fig. 6 Experimental setup.

the $1 \mathrm{MHz}$ central frequency broadband. The transducer is set on the inclined acrylic wedge so that Lamb waves are excited by the refraction of the longitudinal wave propagating through the wedge into the plate specimen. In order to excite the $S_{0}, A_{0}$, and $A_{1}$ modes, the inclination angles of the wedge are chosen as $33^{\circ}, 70^{\circ}$, and $17^{\circ}$, respectively, which are obtained by the Snell's law. The laser vibrometer (Graphtec AT3700 \& AT0023) is used as a receiver to measure the normal velocity on the plate surface. The signal detected by the laser vibrometer is sent to the oscilloscope (Hawlett Packard 54810A) to record the waveform with $0.1 \mu \mathrm{s}$ sampling time at each point. To improve the signal-to-noise ratio, we take the average of 256 successive waveforms. The recorded signals are then sent to the computer for further signal processing.

A steel plate with the material properties of $c_{L}=5940 \mathrm{~m} / \mathrm{s}$ and $c_{T}=3200 \mathrm{~m} / \mathrm{s}$ is used as a specimen in our experiment. The plate has the thickness of $2 \mathrm{~mm}$, the width of $150 \mathrm{~mm}$, and the length of $250 \mathrm{~mm}$. The setup for the measurement of reflected waves is shown in Fig. 6(b). The transducer is set in the direction with the angle $\theta$ to the normal direction of the edge of the plate. To obtain the amplitude of the reflected wave of the same mode with the incident wave, the points of measurement are chosen on the line $A B$ directed with the reflected angle $\theta$. In our experiments, 32 points with equal intervals of $1 \mathrm{~mm}$ are taken on the line $A B$.

The numerical computation is carried out for 2-D problems, but the wave field in the experiment has 3-D configuration. Since there is a difference in attenuations between 2-D and 3-D wave fields, it is necessary to measure the reference signal to remove the effect of the attenuation. The setup for the measurement of the reference wave is shown in Fig. 6(c). The measurements are carried out on the line $A^{\prime} B^{\prime}$, which coincides with the center line of the transducer. The distances $\left|C^{\prime} O^{\prime}\right|,\left|O^{\prime} A^{\prime}\right|$, and $\left|A^{\prime} B^{\prime}\right|$ in Fig. 6(c) are equal to $|C O|,|O A|$, and $|A B|$ in Fig. 6(b), respectively.

The signals obtained by the measurement systems shown in Figs. 6(b) and (c) are Fourier transformed ${ }^{14)}$ with respect to time and measurement positions to obtain the amplitudes $A_{\text {rfl }}(f, k)$ and $A_{\text {ref }}(f, k)$ of the reflected and reference waves, respectively, in the frequency-wavenumber domain. The reflection coefficient $\left|r_{n}^{n}\right|$ is then found as

$$
\left|r_{n}^{n}\right|=\frac{A_{\mathrm{rfl}}\left(f, k_{n}\right)}{A_{\mathrm{ref}}\left(f, k_{n}\right)},
$$

where $k_{n}$ is the wavenumber of the mode $n$ at the frequency $f$. In the calculation of the reflection coefficients in eq. (33), only the central frequency of the 15-cycle-toneburst signal $(f=1 \mathrm{MHz})$ is used, which corresponds to the dimensionless frequency $\omega h / c_{T}=1.96$.

Experimental results of the reflection coefficients $\left|r_{S_{0}}^{S_{0}}\right|$, $\left|r_{A_{0}}^{A_{0}}\right|$, and $\left|r_{A_{1}}^{A_{1}}\right|$ are shown by circles in Figs. 4(b), 5(a), and 5(c), respectively. Some errors between the experiments and the numerical results are observed in these figures. The errors could be attributed by 3 -D effects which are not considered in our 2-D reflection analysis or measurement errors due to the large noise in the laser vibrometer and different tranducerplate contact conditions in the measurements of reflected and references waves. Though there are some errors, fairly good correlation is found between the experiments and the numerical results.

\section{Conclusions}

The method to decompose a wave field in a plate into guided wave modes has been developed by using the generalized orthogonality of guided wave modes. The mode decomposition has been applied to solve the reflection problems of obliquely incident guided waves by an edge of a plate. The comparison between the results of the reflection problems of normal incidence solved by the mode decomposition method and by the previous study of mode-exciting method shows very good agreement. The approximated solutions for the $S_{0}$ and $S H_{0}$ modes incidences in the low frequency range have been derived based on the reflections of $\mathrm{P}$ and SV waves in a half space, respectively. The experiments have also been carried out to obtain the reflected amplitudes of obliquely incident guided waves by the edge of the steel plate. Fairly good correlation was found between the experimental results and the numerical results.

Finally, we give a comment on further study of the present study. The analysis technique presented here can be extended to the edge-reflection analysis of an incident guided wave with nonplanar wavefront, such as the incident wave generated by a transducer. The edge-reflection problem of nonplanar incidence can be decomposed into the reflection problems of plane incident waves by applying the Fourier transform with respect to the axis parallel to the edge. The solution of the edge-reflection problem of nonplanar incidence can further be utilized to clarify the propagation of guided waves in a plate with a finite width. 
This work was supported by Grant-in Aid of the Ministry of Education, Culture, Sports, Science and Technology, and the Japan Society for the Promotion of Science.

\section{REFERENCES}

1) I. A. Viktorov: Rayleigh and Lamb Waves: Physical Theory and Application, (Plenum, New York, 1967).

2) J. D. Achenbach: Wave Propagation in Elastic Solids, (North-Holland, Amsterdam, 1973).

3) B. A. Auld: Acoustic Fields and Waves in Solids, (Wiley, New York, 1973).

4) P. J. Torvik: J. Acous. Soc. Am. 41 (1967) 346-353.
5) B. A. Auld and E. M. Tsao: IEEE Trans. Sonics Ultrason. SU-24 (1977) 317-326.

6) B. Morvan, N. Wilkie-Chancellier, H. Duflo, A. Tinel and J. Duclos: J. Acoust. Soc. Am. 113 (2003) 1417-1425.

7) N. Wilkie-Chancellier, H. Duflo, A. Tinel and J. Duclos: J. Acoust. Soc. Am. 117 (2005) 194-199.

8) R. D. Gregory and I. Gladwell: J. Elast. 13 (1983) 185-206.

9) M. Koshiba: Electron. Lett. 19 (1983) 256-257.

10) J. M. Galan and R. Abascal: Int. J. Numer. Meth. Engng 53 (2002) $1145-1173$.

11) Y. H. Cho and J. L. Rose: J. Acous. Soc. Am. 99 (1996) 2097-2109.

12) A. Gunawan and S. Hirose: J. Acoust. Soc. Am. 115 (2004) 996-1005.

13) N. Wilkie-Chancellier, H. Duflo, A. Tinel and J. Duclos: Ultrason. 42 (2004) 377-381.

14) D. Alleyne and P. Cawley: J. Acoust. Soc. Am. 89 (1991) 1159-1168. 